\title{
Maximal Nontraceable Graphs with Toughness less than One *
}

\author{
Frank Bullock, Marietjie Frick, Joy Singleton’ Susan van Aardt \\ University of South Africa, \\ P.O. Box 392, Unisa, 0003, \\ South Africa. \\ bullofes@unisa.ac.za, frickm@unisa.ac.za \\ singlje@unisa.ac.za, vaardsa@unisa.ac.za
}

\author{
Kieka (C.M.) Mynhardt \\ University of Victoria, \\ P.O. Box 3045 Victoria, BC, \\ Canada V8W 3P4. \\ mynhardt@math.uvic.ca
}

Submitted: Jun 21, 2006; Accepted: Jan 14, 2008; Published: Jan 21, 2008

Mathematics Subject Classification: 05C38

\begin{abstract}
A graph $G$ is maximal nontraceable (MNT) if $G$ does not have a hamiltonian path but, for every $e \in E(\bar{G})$, the graph $G+e$ has a hamiltonian path. A graph $G$ is 1-tough if for every vertex cut $S$ of $G$ the number of components of $G-S$ is at most $|S|$. We investigate the structure of MNT graphs that are not 1-tough. Our results enable us to construct several interesting new classes of MNT graphs.
\end{abstract}

Keywords: maximal nontraceable, hamiltonian path, traceable, nontraceable, toughness

\section{Introduction}

We consider only simple, finite graphs. We denote the vertex set, the edge set, the order and the size of a graph $G$ by $V(G), E(G), v(G)$ and $e(G)$, respectively. The open

*This material is based upon work supported by the National Research Foundation under Grant number 2053752 and Thuthuka Grant number TTK2005081000028.

${ }^{\dagger}$ Corresponding author.

${ }^{\ddagger}$ Visit to University of South Africa (while this paper was written) supported by the Canadian National Science and Engineering Research Council. 
neighbourhood of a vertex $v$ in $G$ is the set $N_{G}(v)=\{x \in V(G): v x \in E(G)\}$. If $N_{G}(v) \cup\{v\}=V(G)$, we call $v$ a universal vertex of $G$. If $U$ is a nonempty subset of $V(G)$ then $\langle U\rangle$ denotes the subgraph of $G$ induced by $U$.

A graph $G$ is hamiltonian if it has a hamiltonian cycle (a cycle containing all the vertices of $G$ ), and traceable if it has a hamiltonian path (a path containing all the vertices of $G)$.

If a graph $G$ has a hamiltonian path with endvertices $x$ and $y$, we say that $G$ is traceable from $x$ to $y$. If $G$ is traceable from each of its vertices, we say that $G$ is homogeneously traceable.

A graph $G$ is maximal nonhamiltonian (MNH) if $G$ is nonhamiltonian, but $G+e$ is hamiltonian for each $e \in E(\bar{G})$, where $\bar{G}$ denotes the complement of $G$.

A graph $G$ is maximal nontraceable (MNT) if $G$ is not traceable, but $G+e$ is traceable for each $e \in E(\bar{G})$.

A noncomplete graph $G$ is $t$-tough if $t \leq|S| / \kappa(G-S)$ for every vertex-cut $S \subset V(G)$, where $\kappa(G-S)$ denotes the number of components in $G-S$ and $t$ is a nonnegative real number. The maximum real number $t$ for which $G$ is $t$-tough is called the toughness of $G$ and is denoted by $t(G)$.

In 1998 Zelinka [14] presented two constructions, which each yielded an infinite class of MNT graphs. We call the graphs in these classes Zelinka graphs, and we call MNT graphs that cannot be constructed by one of Zelinka's constructions non-Zelinka MNT graphs. By consulting [10] we can see that all MNT graphs of order less than 8 are Zelinka graphs. (Zelinka originally conjectured that all MNT graphs can be constructed by his methods, but he later retracted this conjecture.)

All Zelinka graphs have toughness less than one. The first non-Zelinka MNT graphs constructed are all 1-tough. (Claw-free, 2-connected ones are presented in [2] and cubic ones in [7].) However, not all MNT graphs with toughness less than one are Zelinka graphs, because Dudek, Katona and Wojda [6] recently constructed an infinite class of non-Zelinka MNT graphs that have cut-vertices and hence have toughness at most 1/2. We shall call these graphs DKW graphs.

In this paper we investigate the structure of MNT graphs with toughness less than 1. Our results enable us to construct several new classes of MNT graphs with toughness less than 1. For example, we construct an infinite family of non-Zelinka MNT graphs having two cut-vertices and three blocks, with the middle block being hamiltonian. (The DKW graphs also have three blocks and 2 cut-vertices, but in their case the middle block is $\mathrm{MNH})$. We also construct an infinite family of non-Zelinka MNT graphs with only two blocks. Among these is a graph of order 8 and size 15. This turns out to be a non-Zelinka MNT graph of smallest possible order and size. Finally, we construct infinite families of 2-connected non-Zelinka MNT graphs with toughness less than 1. 


\section{The Zelinka Constructions}

The constructions given by Zelinka [14] provide two important classes of MNT graphs with toughness less than one. We describe the constructions briefly.

\section{Zelinka Type I graphs}

Suppose $p$ is a non-negative integer and $a_{1}, \ldots, a_{k}$, where $k=p+2$, are positive integers. Let $U_{0}, U_{1}, \ldots, U_{k}$ be pairwise disjoint sets of vertices such that $\left|U_{0}\right|=p$ and $\left|U_{i}\right|=a_{i}$ for $i=1, \ldots, k$. Let the graph $G$ have $V(G)=\bigcup_{i=0}^{k} U_{i}$ and $E(G)$ be such that the induced subgraphs $\left\langle U_{0} \cup U_{i}\right\rangle$ for $i=1, \ldots, k$ are complete graphs. We call such a graph $G$ a Zelinka Type I graph.

This construction is represented diagrammatically in Figure 1.

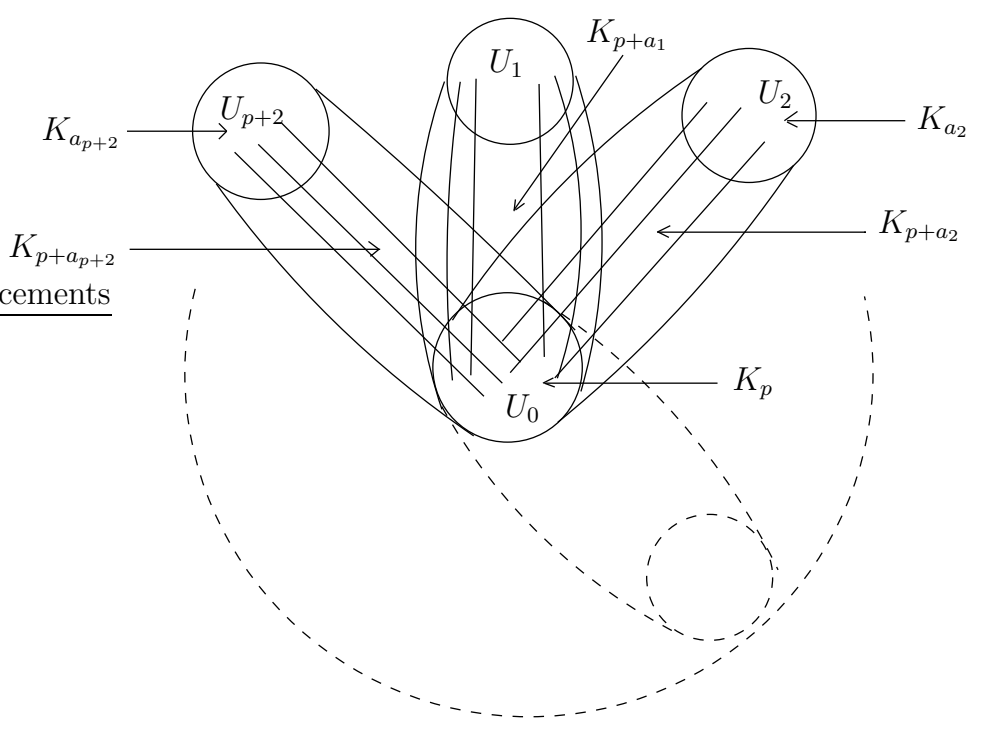

Figure 1: Zelinka Type I graph

\section{Zelinka Type II graphs}

Suppose $p, q, r, a_{1}, \ldots, a_{p}, b_{1}, \ldots, b_{q}, c_{1}, \ldots, c_{r}$ are positive integers and $s$ a non-negative integer.

Let $U_{0}, U_{1}, \ldots, U_{p}, V_{0}, V_{1}, \ldots, V_{q}, W_{0}, W_{1}, \ldots, W_{r}, X$ be pairwise disjoint sets of vertices such that $\left|U_{0}\right|=p,\left|U_{i}\right|=a_{i}$ for $i=1, \ldots, p,\left|V_{0}\right|=q,\left|V_{i}\right|=b_{i}$ for $i=1, \ldots, q,\left|W_{0}\right|=r,\left|W_{i}\right|=c_{i}$ for $i=1, \ldots, r$ and $|X|=s$.

Let the graph $G$ have $V(G)=\left(\bigcup_{i=0}^{p} U_{i}\right) \cup\left(\bigcup_{i=0}^{q} V_{i}\right) \cup\left(\bigcup_{i=0}^{r} W_{i}\right) \cup X$ and $E(G)$ be such that the induced subgraphs $\left\langle U_{0} \cup U_{i}\right\rangle$ for $i=1, \ldots, p,\left\langle V_{0} \cup V_{i}\right\rangle$ for $i=1, \ldots, q,\left\langle W_{0} \cup W_{i}\right\rangle$ for $i=1, \ldots, r$, and $\left\langle U_{0} \cup V_{0} \cup W_{0} \cup X\right\rangle$ are all complete graphs. We call such a graph $G$ a Zelinka Type II graph.

This construction is represented diagrammatically in Figure 2. 


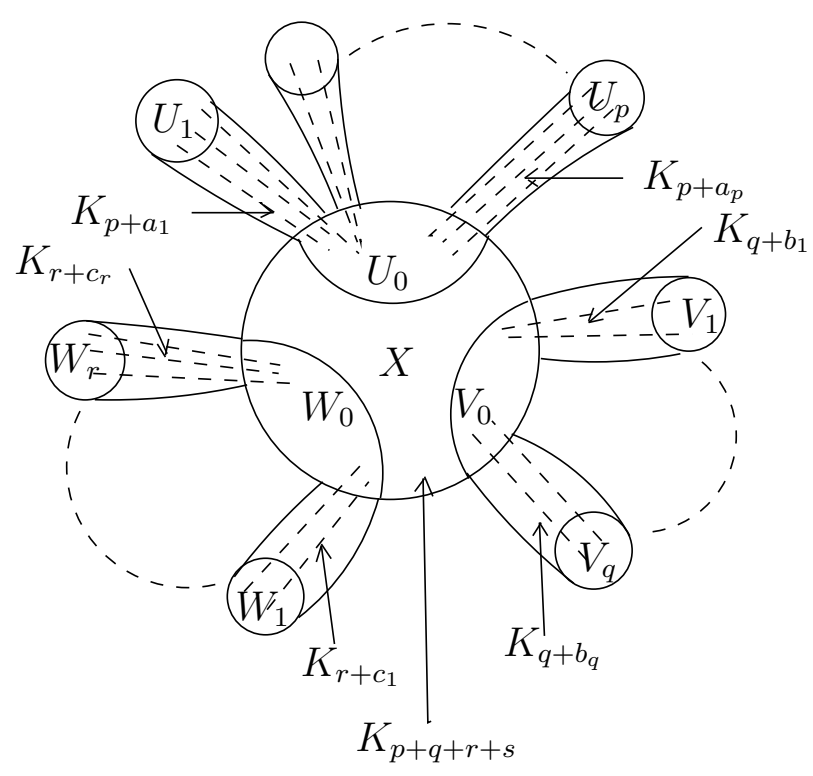

Figure 2: Zelinka Type II graph

Remark 2.1 By consulting [10] we see that all MNT graphs with fewer than 8 vertices are Zelinka graphs.

If $G$ is the graph in Figure 1, then $\kappa\left(G-U_{0}\right)=\left|U_{0}\right|+2$, while if $G$ is the graph in Figure 2, then $\kappa\left(G-U_{0}\right)=\left|U_{0}\right|+1$. Thus all Zelinka graphs have toughness less than one.

\section{Maximal nontraceable graphs with toughness less than one}

Suppose $P$ is a path in a graph $G$, with endvertices $a$ and $z$. If we regard $P$ as going from $a$ to $z$, we denote it by $P[a, z]$, and if we reverse the direction we denote it by $P[z, a]$. If $u, v \in V(P)$, then $P[u, v]$ denotes the subpath of $P$ that starts at $u$ and ends at $v$, and $P(u, v)=P[u, v]-\{u, v\}$.

If a graph $G$ has two vertex disjoint paths, $F^{1}$ and $F^{2}$, such that $V(G)=V\left(F^{1}\right) \cup$ $V\left(F^{2}\right)$, then $F^{1}, F^{2}$ is called a 2-path cover of $G$.

If $G$ is an MNT graph with $t(G)<1$, then it is easy to see that $G$ has a vertex-cut $S$ such that $\kappa(G-S)=|S|+2$ or $\kappa(G-S)=|S|+1$. We now characterize the first of these two cases.

Theorem $3.1 G$ is an MNT graph having a subset $S$ such that $\kappa(G-S)=|S|+2$ if and only if $G$ is a Zelinka Type I graph.

Proof. Let $G$ be a Zelinka Type I graph as depicted in Figure 1. Then $\kappa\left(G-U_{0}\right)=\left|U_{0}\right|+2$. 
Conversely, suppose that $\kappa(G-S)=k=|S|+2$ and $A_{1}, A_{2}, \ldots, A_{k}$ are the $k$ components of $G-S$. Suppose that for some $i$ the graph $\left\langle S \cup A_{i}\right\rangle$ has two nonadjacent vertices, $u$ and $v$. Then $S$ is a vertex-cut of $G+u v$ and $(G+u v)-S$ has $|S|+2$ components. But then $G+u v$ is not traceable. This contradiction proves that $\left\langle S \cup A_{i}\right\rangle$ is complete for $i=1,2, \ldots, k$, and hence $G$ is a Zelinka Type I graph.

If $G$ is a Zelinka Type II graph as depicted in Figure 2, then $\kappa\left(G-U_{0}\right)=\left|U_{0}\right|+1$ and every component of $G-U_{0}$ except for one is complete. We suspected at first that the Zelinka Type II graphs are the only ones with this property. However, the following theorem enabled us to find non-Zelinka graphs with this property.

\subsection{The case where $G-S$ has one noncomplete component}

Theorem 3.2 Let $G$ be a connected graph with a minimum vertex-cut $S$ such that $|S|=k$ and $G-S$ has $k+1$ components $G_{1}, G_{2}, \ldots, G_{k}, H$, all of which are complete except for $H$. Then $G$ is $M N T$ if and only if the following conditions hold:

(i) $\left\langle S \cup V\left(G_{i}\right)\right\rangle$ is complete, for $i=1,2, \ldots, k$.

(ii) $H$ is traceable from each vertex in $V(H)-N_{H}(S)$.

(iii) $H$ is not traceable from any vertex in $N_{H}(S)$, but for every pair $u, v$ of nonadjacent vertices in $H$, the graph $H+u v$ is traceable from a vertex in $N_{H}(S)$.

(iv) Every vertex in $S$ is adjacent to every vertex in $N_{H}(S)$.

(v) For every $a \in N_{H}(S)$ the graph $H$ has a 2-path cover $F^{1}[a, b], F^{2}[c, d]$ where $d \in$ $N_{H}(S) ; b, c \in V(H)$.

Proof. Suppose $G$ is MNT. We show that $G$ satisfies (i) - (v).

(i) If $x, y \in S$ such that $x y \notin E(G)$, then any path in $G+x y$ containing $x y$ contains vertices from at most $k$ components of $G-S$, which implies that $G+x y$ has no hamiltonian path. This contradiction implies that $\langle S\rangle$ is a complete graph. Now suppose that for some $j \in\{1, \ldots, k\}$ there is a vertex $x \in S$ and a vertex $v \in V\left(G_{j}\right)$ such that $x v \notin E(G)$. Let $P$ be a hamiltonian path of $G+x v$. Since $\kappa(G-S)=$ $|S|+1$, the path $P$ visits each component of $G_{j}$ exactly once. If $P$ has an endvertex in $G_{j}$, then $P$ has a subpath containing all the vertices of $G-V\left(G_{j}\right)$, ending in $x$. But then, since $x$ is adjacent to some vertex in $G_{j}$ and $G_{j}$ is complete, $G$ is traceable, a contradiction. We may therefore assume that $k \geq 2$ and $P$ has a subpath $x P[v, w] y$ such that $y \in S$ and $P[v, w]$ is a hamiltonian path of $G_{j}$. If $N_{G_{j}}(x) \cup N_{G_{j}}(y)=\{w\}$, then $(S-\{x, y\}) \cup\{w\}$ is a vertex-cut of $G$, contradicting the minimality of $S$. Hence $G_{j}$ has two distinct vertices, $u$ and $z$ such that $x u, z y \in E(G)$. Since $G_{j}$ is complete, $G_{j}$ has a hamiltonian path $Q[u, z]$. If in $P$ we replace the path $P[v, w]$ with the path $Q[u, z]$, we obtain a hamiltonian path of $G$, a contradiction. This proves that $\left\langle S \cup V\left(G_{i}\right)\right\rangle$ is complete, for $i=1,2, \ldots, k$. 
(ii) Let $v \in V(H)-N_{H}(S)$ and $x \in S$. Then $G+x v$ has a hamiltonian path $P$. Since $P$ visits $H$ only once, $H$ is traceable from $v$.

(iii) It follows from (i) that $G-V(H)$ is homogeneously traceable. Hence, $H$ is not traceable from any vertex in $N_{H}(S)$, otherwise $G$ would be traceable. If $u, v \in$ $V(H)$, then $G+u v$ has a hamiltonian path which visits $H$ only once, and hence $H+u v$ is traceable from a vertex in $N_{H}(S)$.

(iv) If there exists a vertex $u \in N_{H}(S)$ and $x \in S$ such that $u x \notin E(G)$, then $G+u x$ has a hamiltonian path, which implies that $H$ is traceable from $u$, contradicting (iii).

(v) Let $a \in\left\langle N_{H}(S)\right\rangle$ and let $v \in G_{1}$. Then $G+a v$ has a hamiltonian path $P$. Since $H$ is not traceable from $a$, it follows that $P$ visits $H$ more than once. Hence, since $\kappa(G-S)=k+1$, it follows that $P$ visits $H$ exactly twice. Thus $H$ has a 2-path cover $F^{1}[a, b], F^{2}[c, d]$ where $d \in N_{H}(S) ; b, c \in V(H)$.

To prove the converse, suppose $G$ satisfies (i) - (v). If $G$ is traceable, then our assumption that $|S|=k$ and $\kappa(G-S)=k+1$ implies that any hamiltonian path of $G$ visits each component of $G-S$ exactly once and that the endvertices of the path are in two different components of $G-S$. Thus $H$ is traceable from a vertex in $N_{H}(S)$. This contradicts (iii). Hence $G$ is not traceable. However, it follows from (i) that $G-V(H)$ is homogeneously traceable.

To show that $G$ is MNT we need to show that $G+u v$ is traceable for all $u, v \in V(G)$, where $u v \notin E(G)$.

Case 1. $u, v \in V(H)$ :

It follows from (i) and (iii) that $G+u v$ is traceable.

Case 2. $u \in V(H), v \in S$ :

By (iv) $u \in V(H)-N_{H}(S)$; hence it follows from (i) and (ii) that $G+u v$ is traceable.

Case 3. $u \in V(H)-N_{H}(S), v \in V\left(G_{i}\right), i=1, \ldots, k$ :

According to (i) and (ii) $G+u v$ is traceable.

Case 4. $u \in N_{H}(S), v \in V\left(G_{i}\right), i=1, \ldots, k$ :

It follows from (i) that $G-V(H)$ has a hamiltonian path $P[x, v]$, where $x \in S$. By (v), $H$ has a 2-path cover $F^{1}[u, b], F^{2}[c, d]$, where $d \in N_{H}(S) ; b, c \in V(H)$. The path $F^{1}[c, d] P[x, v] F^{2}[u, b]$ is a hamiltonian path of $G$.

Case 5. Consider $k \geq 2$. Let $u \in V\left(G_{i}\right)$ and $v \in V\left(G_{j}\right), i \neq j, i, j=1, \ldots, k$ :

It follows from (i) that $(G+u v)-V(H)$ has a hamiltonian path $P[x, y]$, where $x, y \in S$. By (vi), $H$ has a 2-path cover $F^{1}[a, b], F^{2}[c, d]$ where $a, d \in N_{H}(S) ; b, c \in V(H)$. Thus $F^{2}[c, d] P[x, y] F^{1}[a, b]$ is a hamiltonian path of $G+u v$.

The following corollary is useful when attempting to construct MNT graphs having the structure described in Theorem 3.2. 
Corollary 3.3 Let $G$ be an MNT graph that has the structure as described in Theorem 3.2. Then the noncomplete component $H$ has no universal vertices.

Proof. Suppose $b$ is a universal vertex of $H$.

If $b \in N_{H}(S)$ then, by (v), $H$ has a 2-path cover $F^{1}[a, b], F^{2}[c, d]$, where $d \in$ $N_{H}(S) ; a, c \in V(H)$. But then, since $b c \in E(H)$, the path $F^{1}[a, b] F^{2}[c, d]$ is a hamiltonian path of $H$ with endvertex $d \in N_{H}(S)$, contradicting (iii).

If $b \notin N_{H}(S)$, then, by (ii), $H$ has a hamiltonian path $Q[b, z]$, for some $z \in V(H)$. Since $z b \in E(H)$, it then follows that $H$ has a hamiltonian cycle. But then $H$ is homogeneously traceable, contradicting (iii).

Remark 3.4 Suppose $G$ is an MNT graph that has the structure as described in Theorem 3.2. Then either every vertex in $S$ is a universal vertex of $G$ and $H$ is $M N T$, or no vertex in $S$ is a universal vertex of $G$ and $H$ is traceable (from every vertex in $\left.V(H)-N_{H}(S)\right)$. We shall present examples of both cases.

If each cut-vertex of a graph $G$ lies in exactly two blocks of $G$, we say that $G$ has a linear block structure. We now show that Theorem 3.2 applies to every MNT graph with a linear block structure.

Lemma 3.5 Suppose $G$ is a connected MNT graph with a cut-vertex $x$ such that $G-x$ has exactly two components. Then exactly one of the two components is a complete graph.

Proof. Let $A$ and $B$ be the components of $G-x$. Then $A$ and $B$ cannot both be complete, otherwise $G$ would be traceable. Suppose $A$ is not complete and let $u, v$ be two nonadjacent vertices in $A$. Then, since $G+u v$ is traceable, $B$ is traceable from $x$. If $B$ is also not complete, then $A$ is also traceable from $x$. But then $G$ is traceable.

Corollary 3.6 Suppose $G$ is an MNT graph with a linear block structure. Then $G$ either has only two blocks, of which exactly one is complete, or $G$ has exactly two cut-vertices and three blocks, in which case the two end-blocks are complete and the middle block is not complete.

Proof. Apply Theorem 3.2(i) to each cut-vertex of G.

Let $G$ be an MNT graph with exactly two blocks. Denote the noncomplete block by $B$, the cut-vertex by $x$ and let $H=B-x$. By Corollary 3.3, $H$ has no universal vertices. By Remark 3.4, either $x$ is a universal vertex of $G$ and $H$ is MNT, or $H$ is traceable, but not from $N_{H}(x)$.

Every Zelinka Type II graph, in which $p=1, q \geq 2, r \geq 2$, is an MNT graph with exactly two blocks, in which the cut-vertex $x$ is not a universal vertex. The smallest such graph is depicted in Figure 3. 


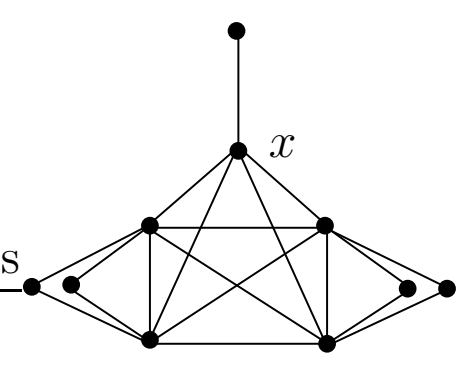

Figure 3: Smallest Zelinka MNT graph with two blocks

We now present non-Zelinka MNT graphs with this property.

Example 3.7 The tarantula, depicted in Figure 4, is a non-Zelinka graph with exactly two blocks, in which the cut-vertex $x$ is not a universal vertex. We note that in the tarantula both $\left\langle x, u_{1}, u_{2}, u_{3}\right\rangle$ and $\left\langle x, w_{1}, w_{2}, w_{3}\right\rangle$ are complete graphs.

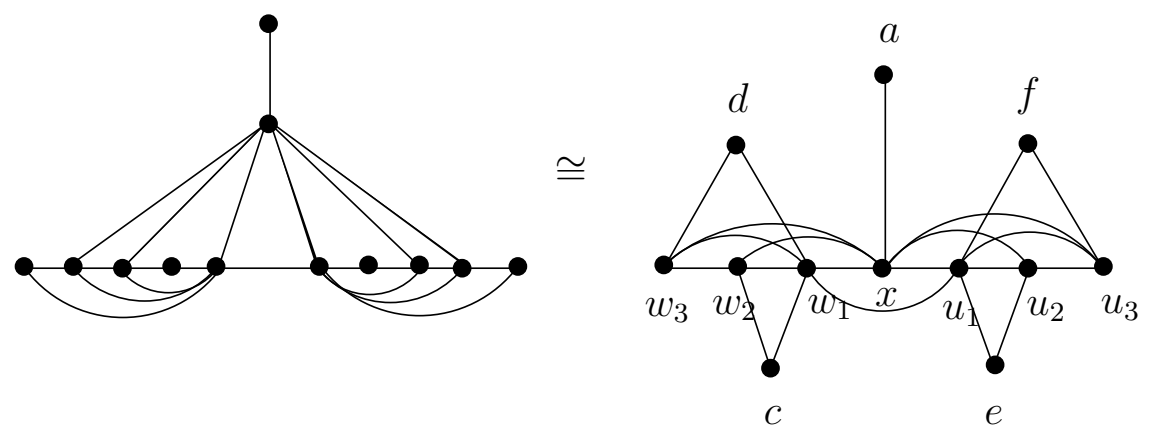

Figure 4: Tarantula

We generalize the tarantula as depicted in Figure 5.

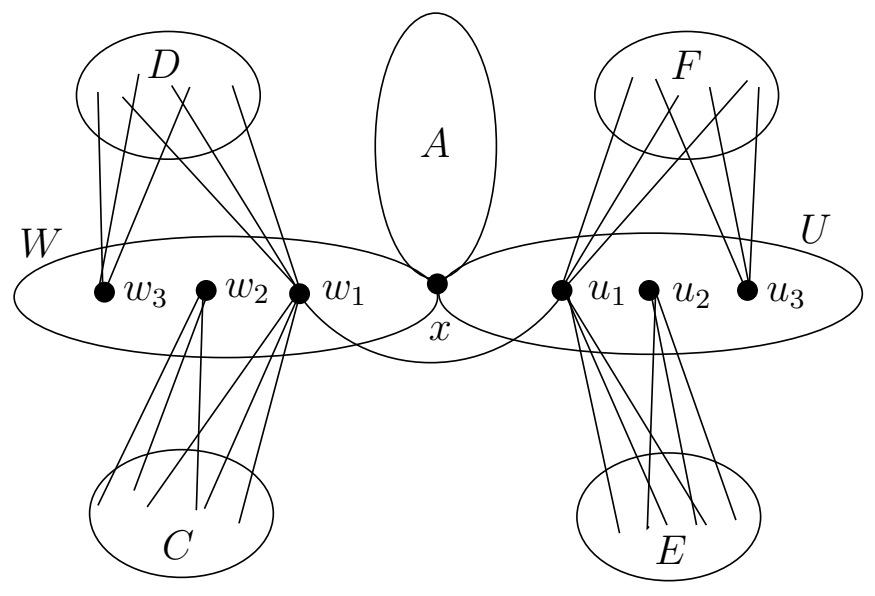

Figure 5: Generalized tarantula 
A generalized tarantula contains three complete graphs, $A$ (of order at least 2), $W$ and $U$ (both of order at least 4 ) which share a single common vertex $x$, and four mutually disjoint complete graphs, $C, D, E$ and $F$ which have no vertices in common with $V(A) \cup$ $V(W) \cup V(U)$. The subgraph $W$ has three distinguished vertices $w_{1}, w_{2}, w_{3}$ and $U$ has three distinguished vertices $u_{1}, u_{2}, u_{3}$. The graph has the following additional adjacencies: $w_{1}$ and $w_{2}$ are adjacent to all vertices in $C, w_{1}$ and $w_{3}$ are adjacent to all vertices in $D$, $u_{1}$ and $u_{2}$ are adjacent to all vertices in $E, u_{1}$ and $u_{3}$ are adjacent to all vertices in $F$, and $w_{1}$ is adjacent to $u_{1}$.

It is easy to check that generalized tarantulas satisfy the conditions of Theorem 3.2. Thus we have an infinite family of non-Zelinka MNT graphs with exactly two blocks, in which the cut-vertex is not a universal vertex.

Next we present MNT graphs with exactly two blocks, in which the cut-vertex is a universal vertex.

Example 3.8 The propeller, shown in Figure 6, is an MNT graph with two blocks, in which the cut-vertex $x$ is a universal vertex. Let $B$ denote the noncomplete block of the propeller. Then $H=B-x$ is the net, which is the smallest MNT graph without universal vertices. Since all MNT graphs of order less than 8 are Zelinka graphs, the propellor is a non-Zelinka MNT graph of smallest order. We do not know of any other non-Zelinka MNT graph of order 8 .
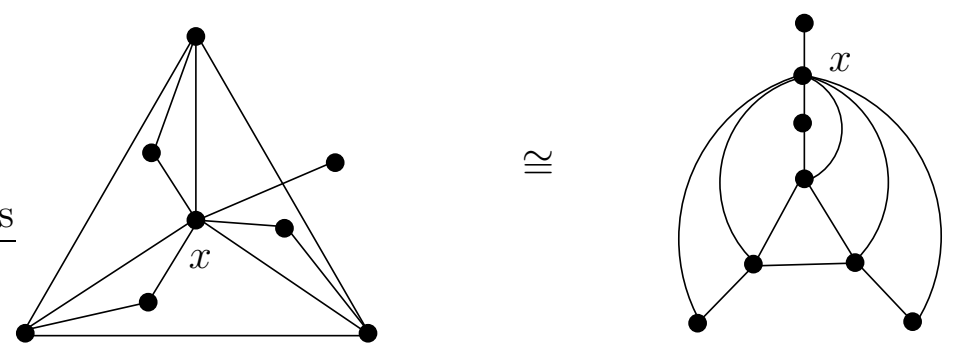

Figure 6: The propeller, a non-Zelinka MNT graph of smallest order

The noncomplete block $B$ of the propeller can also be described as the graph obtained from a $K_{4}$ by subdividing the three edges incident with a fixed vertex $x$ and then adding the relevant edges to make $x$ a universal vertex. This description allows us to generalize the propellor to obtain an MNT graph of order $n \geq 8$, as depicted in Figure 7. We let $A$ be a complete graph of arbitrary order, and for $B$ we replace the three triangles incident with $x$ with complete graphs of arbitrary order. 


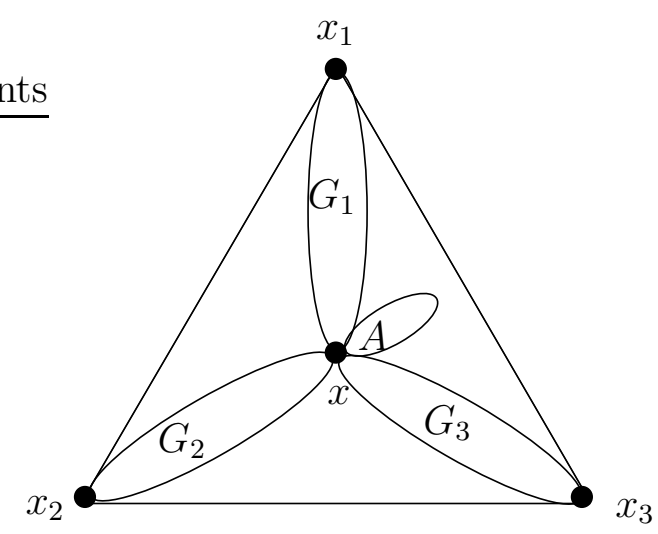

Figure 7: A generalized propeller

The construction given above can be further generalized by starting with any $K_{n}$, with $n \geq 5$, instead of $K_{4}$, and replacing any three edges incident with $x \in V\left(K_{n}\right)$ with complete graphs.

It follows directly from Theorem 3.2 that the generalized propellers are MNT.

Now suppose $G$ is an MNT graph with exactly three blocks, $B_{1}, B$ and $B_{2}$ and two cut-vertices, $x$ and $y$, with $B$ being the middle block and $x \in V\left(B_{1}\right) \cap V(B)$ and $y \in V\left(B_{2}\right) \cap V(B)$. Then, obviously, $x y \in E(G)$ and, by Corollary $3.6 B_{1}$ and $B_{2}$ are complete graphs, while $B$ is not complete. Moreover, it is obvious that $B$ does not have a hamiltonian path with endvertices $x$ and $y$, but, for any $e \in E(\bar{G})$ the graph $G+e$ has such a hamiltonian path. This implies that either the middle block $B$ is MNH, or $B$ is hamiltonian but no hamiltonian cycle contains the edge $x y$.

Every Zelinka Type II graph with $p=q=1, r \geq 2$ is an MNT graph with two cut-vertices and three blocks, in which the middle block is MNH. The smallest such graph is depicted in Figure 8.

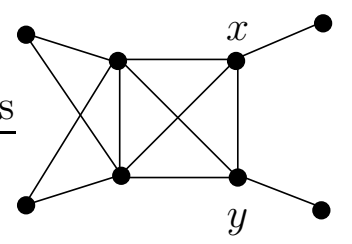

Figure 8: Smallest Zelinka MNT graph with three blocks

As shown in the next example, the middle block $B$ may be chosen from various $\mathrm{MNH}$ graphs to produce non-Zelinka MNT graphs with three blocks and two cut-vertices.

Example 3.9 (Dudek, Katona and Wojda [6]):

Consider a cubic MNH graph $B$ with the properties that 
$\mathrm{D}(1): \quad$ there is an edge $x y$ of $B$, such that $N(x) \cap N(y)=\emptyset$, and

$\mathrm{D}(2): \quad B+e$ has a hamiltonian cycle containing $x y$ for every $e \in E(\bar{B})$.

Take two graphs $B_{1}$ and $B_{2}$, with $B_{1} \cong K_{1}$ and $B_{2} \cong K_{1}$ or $B_{2} \cong K_{2}$ and join each vertex of $B_{1}$ to $x$ and each vertex of $B_{2}$ to $y$. The resulting graph, which we call a DKW-graph, is an MNT graph with three blocks and two cut-vertices.

In [4], [5] and [8] constructions of cubic MNH graphs with properties $\mathrm{D}(1)$ and $\mathrm{D}(2)$ are given. It transpires that such graphs of order $n$ exist for each even $n \geq 52$ and $n \in I=\{10,20,28,36,38,40,44,46,48\}$. This yields DKW-graphs of order $n$ for every $n \geq 54$ and each $n=i+2$ or $n=i+3$, where $i \in I$. It is shown in [8] that every MNT graph of order $n \geq 10$ has size at least $\left\lceil\frac{3 n-2}{2}\right\rceil$. The DKW graphs realise this lower bound.

By consulting [10] we see that the Petersen graph is the smallest cubic MNH graph satisfying $\mathrm{D}(1)$ and $\mathrm{D}(2)$.

Figure 9 depicts a DKW-graph constructed from the Petersen graph.

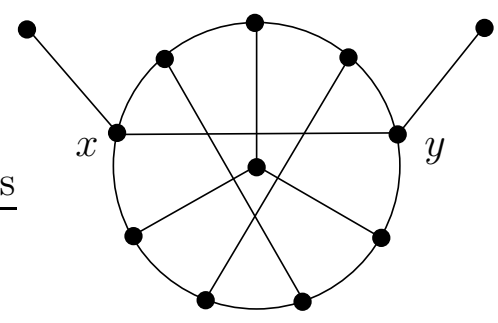

Figure 9: An MNT graph with the Petersen graph as the middle block

DKW-graphs can be generalized by replacing each of $B_{1}$ and $B_{2}$ with an arbitrarily large complete graph. Each such graph is an MNT graph with exactly three blocks and two cut-vertices, in which the middle block is MNH.

Next we present MNT graphs with 3 blocks and 2 cut-vertices in which the middle block is hamiltonian.

Example 3.10 The sputnik, depicted in Figure 10(a), is a smallest MNT graph that has exactly three blocks and two cut-vertices in which the middle block is hamiltonian. (It is proved in [11] that no such graph has order less than 10 and that every MNT graph of order 10 has at least 15 edges.)

By replacing each triangle as well as each end block of the sputnik by a complete graph of arbitrary order, in the manner shown in Figure 10(b), we obtain, for every $n \geq 10$, an MNT graph of order $n$ that has three blocks and two cut-vertices in which the middle block is hamiltonian. We call such a graph a generalized sputnik. 


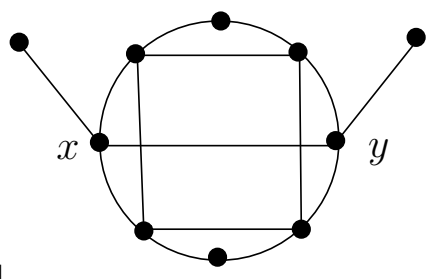

(a)

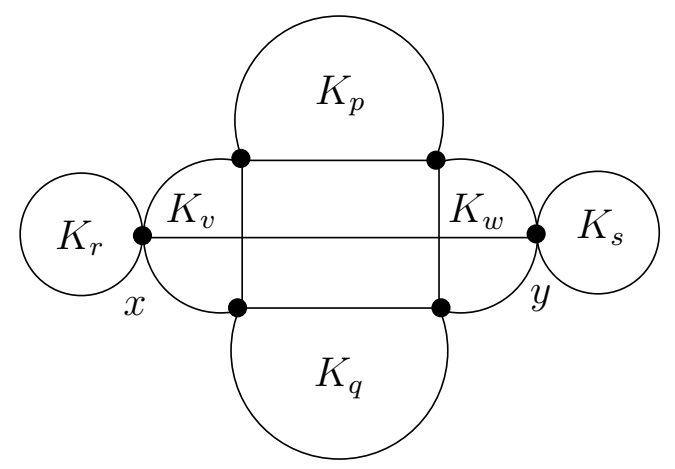

(b)

Figure 10: The sputnik and a generalized sputnik

\subsection{The case where $G-S$ has more than one noncomplete com- ponent}

There also exist MNT graphs having a subset $S$ such that $|S|=k$ and $\kappa(G-S)=k+1$ such that more than one component of $G-S$ is noncomplete. By Theorem 3.2(i), such graphs have no cut-vertices, i.e. they are 2-connected. We consider here only those that have connectivity equal to 2 . We first prove the following.

Lemma 3.11 Suppose $G$ is a graph with a minimal vertex-cut $S$ such that $|S|=2$ and $G-S$ has three components $G_{1}, G_{2}, G_{3}$. If $G$ is MNT then either exactly two of the components are complete or none of the components is complete.

Proof. Let $S=\left\{x_{1}, x_{2}\right\}$. If all three components are complete then $G$ is traceable.

Assume that exactly one of the components, say $G_{3}$, is complete. Then, as in the proof of the first part of (i) in Theorem 3.2, the graph $\left\langle S \cup V\left(G_{3}\right)\right\rangle$ is complete. Now not both $\left\langle V\left(G_{1}\right) \cup\left\{x_{i}\right\}\right\rangle$ and $\left\langle V\left(G_{2}\right) \cup\left\{x_{j}\right\}\right\rangle,\{i, j\}=\{1,2\}$ are traceable, respectively, from $x_{i}$ and $x_{j}$, otherwise $G$ is traceable.

Suppose $u, v \in V\left(G_{1}\right)$ and $u v \notin E(G)$. Then, since $G+u v$ is traceable,

(i) $\left\langle V\left(G_{1}\right) \cup\left\{x_{1}\right\}\right\rangle+u v$ is traceable from $x_{1}$ and $\left\langle V\left(G_{2}\right) \cup\left\{x_{2}\right\}\right\rangle$ is traceable from $x_{2}$; or

(ii) $\left\langle V\left(G_{1}\right) \cup\left\{x_{2}\right\}\right\rangle+u v$ is traceable from $x_{2}$ and $\left\langle V\left(G_{2}\right) \cup\left\{x_{1}\right\}\right\rangle$ is traceable from $x_{1}$.

Without loss of generality we assume the first case is true. Now suppose $z, w \in V\left(G_{2}\right)$ and $z w \notin E(G)$. Then $\left\langle V\left(G_{2}\right) \cup\left\{x_{1}\right\}\right\rangle+z w$ is traceable from $x_{1}$ and $\left\langle V\left(G_{1}\right) \cup\left\{x_{2}\right\}\right\rangle$ is traceable from $x_{2}$. (If $\left\langle V\left(G_{1}\right) \cup\left\{x_{1}\right\}\right\rangle$ was traceable from $x_{1}$, then $G$ would be traceable.) So $\left\langle V\left(G_{i}\right) \cup\left\{x_{2}\right\}\right\rangle, i=1,2$ is traceable from $x_{2}$. Also, $G_{i}$ is not traceable from any vertex in $N_{i}\left(x_{1}\right)=N_{G}\left(x_{1}\right) \cap V\left(G_{i}\right)$ for $i=1,2$. Consider $G+u v$, where $u \in N_{1}\left(x_{1}\right)$ and 
$v \in N_{2}\left(x_{1}\right)$. Since $G_{1}$ and $G_{2}$ are not traceable, respectively, from $u$ and $v$, a hamiltonian path in $G+u v$ visits each of $G_{1}$ and $G_{2}$ at least twice and $G_{3}$ at least once and this is impossible.

Zelinka Type II graphs with $p=2, q \geq 2$ and $r \geq 2$ are MNT graphs satisfying the conditions of Lemma 3.11 with exactly two of the components of $G-S$ being complete. (Take $S=U_{0}$, for example).

We now investigate the case where $G$ is an MNT graph satisfying the conditions of Lemma 3.11 in which all three components of $G-S$ are noncomplete. We first consider the case where $x$ and $y$ have disjoint neighbourhoods, and then we consider a case where $x$ and $y$ have the same open neighbourhood. At present we know of no examples in which $N_{G_{i}}(x)$ and $N_{G_{i}}(y)$ are neither disjoint nor equal for some $i$.

Theorem 3.12 Let $G$ be a graph with a minimal vertex-cut $S=\{x, y\}$ such that $G-S$ consists of three noncomplete components $G_{1}, G_{2}, G_{3}$ and $N_{G_{i}}(x) \cap N_{G_{i}}(y)=\emptyset$, for $i=$ $1,2,3$. Let $X_{i}=\left\langle V\left(G_{i}\right) \cup\{x, y\}\right\rangle$.

Then $G$ is maximal nontraceable if and only if the following hold.

(i) $x y \in E(G)$.

(ii) For each $i=1,2,3$ there is no hamiltonian cycle in $X_{i}$ containing the edge $x y$, but $X_{i}+e$ has a hamiltonian cycle containing $x y$ for each $e \in E\left(\overline{X_{i}}\right)$.

Proof. Suppose $G$ is MNT.

(i) If $x y \notin E(G)$, then a longest path in $G+x y$ contains vertices from at most two components of $G-S$, and hence $G+x y$ is nontraceable.

(ii) Suppose, without loss of generality, that $X_{2}$ has a hamiltonian cycle containing $x y$. Let $e \in E\left(\overline{G_{2}}\right)$. Since $G+e$ is traceable there is a hamiltonian path in $X_{1}-y$ with endvertex $x$ and a hamiltonian path in $X_{3}-x$ with endvertex $y$. Since $X_{2}$ has a hamiltonian path with endvertices $x$ and $y$, it follows that $G$ is traceable, a contradiction. Thus no $X_{i}$ has a hamiltonian cycle containing $x y$. Now consider $G+e$, where $e \in E\left(\overline{X_{i}}\right)$. Then a hamiltonian path in $G+e$ contains a hamiltonian path in $X_{i}+e$ with endvertices $x$ and $y$, and thus a hamiltonian cycle containing $x y$.

To prove the converse, suppose $G$ satisfies conditions (i) and (ii). If $G$ were traceable, then some $X_{i}$ would contain a hamiltonian path with endvertices $x$ and $y$ and hence $X_{i}$ would contain a hamiltonian cycle containing the edge $x y$, a contradiction. Thus $G$ is nontraceable.

Since $S$ is a minimal vertex-cut of $G$ it follows that $x$ and $y$ each have at least one neighbour in each $G_{i}, i=1,2,3$. Hence, since $N_{G_{i}}(x) \cap N_{G_{i}}(y)=\emptyset$, there is at least one vertex in $G_{i}$ not adjacent to $x$ and at least one vertex in $G_{i}$ not adjacent to $y$. Suppose $v \in V\left(G_{i}\right)$ is not adjacent to $x$. Then, by (ii), $X_{i}+v x$ has a hamiltonian cycle containing 
$v x y$, so $G_{i}$ has a hamiltonian path with $v$ and $w$ as endvertices, where $w$ is adjacent to $y$. Similarly, if $u \in V\left(G_{i}\right)$ is not adjacent to $y$, then $G_{i}$ has a hamiltonian path with $u$ and $z$ as endvertices, where $z$ is adjacent to $x$. Thus each $G_{i}$ has two hamiltonian paths, one with endvertex adjacent to $x$ and the other with endvertex adjacent to $y$.

We now prove that $G+u v$ is traceable for $u, v \in V(G)$ and $u v \notin E(G)$.

Case 1. $u, v \in V\left(X_{i}\right)$, say $i=2$.

Then $G+u v$ has a hamiltonian path obtained from a hamiltonian path in $G_{1}$ with one endvertex adjacent to $x$, followed by a hamiltonian path in $X_{2}+u v$ with endvertices $x$ and $y$, followed by a hamiltonian path in $G_{3}$ with endvertex adjacent to $y$.

Case 2. $u \in V\left(G_{i}\right), v \in V\left(G_{j}\right), i \neq j$, say $i=1$ and $j=2$.

Suppose, without loss of generality, that $v$ is not adjacent to $x$. Since $u$ is nonadjacent to $x$ or $y$, it follows from condition (ii) that $G_{1}$ has a hamiltonian path ending at $u$. Also, $G_{2}$ has a hamiltonian path $P$ with one endvertex $v$ and the other endvertex adjacent to $y$. Then $G+u v$ has a hamiltonian path obtained from a hamiltonian path in $G_{1}$ with endvertex $u$, followed by $P$, then $y, x$, followed by a hamiltonian path in $G_{3}$ with endvertex adjacent to $x$.

It follows from condition (ii) of the above theorem that each graph $X_{i}, i=1,2,3$ is either hamiltonian (but no hamiltonian cycle contains the edge $x y$ ) or MNH. We present two examples of graphs that satisfy the conditions of Theorem 3.12, the first in which all the graphs $X_{i}$ are MNH (see Figure 11) and the second in which all the graphs $X_{i}$ are hamiltonian (see Figure 12). Note that in Figure 11 each $X_{i}=\left\langle V\left(G_{i}\right) \cup\{x, y\}\right\rangle$ is isomorphic to the Petersen graph and in Figure 12 each $X_{i}=\left\langle V\left(G_{i}\right) \cup\{x, y\}\right\rangle$ is isomorphic to the middle block of the sputnik.

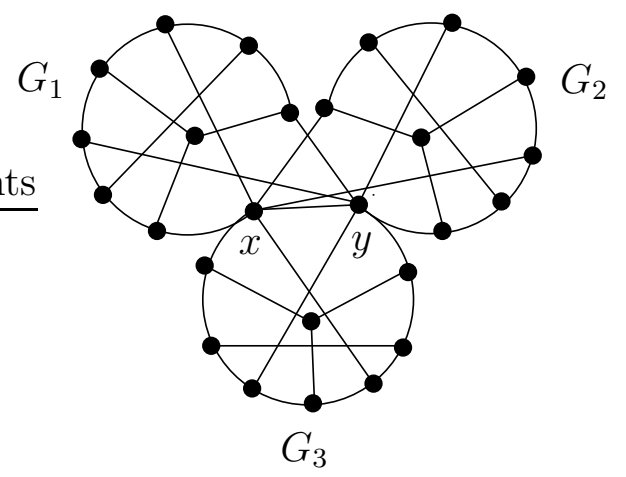

Figure 11: First example of graph satisfying the conditions of Theorem 3.12 


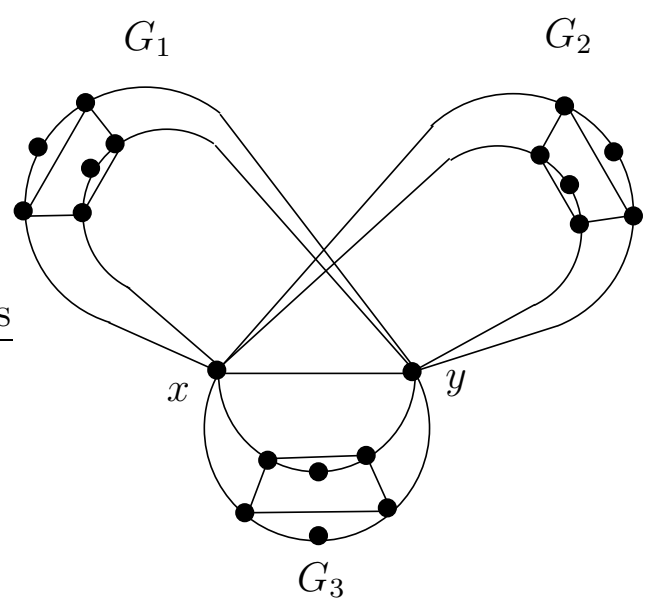

Figure 12: Second example of graph satisfying the conditions of Theorem 3.12

A graph $G$ is hamiltonian-connected (Hc) if for every two distinct vertices $x, y \in V(G)$ there is a hamiltonian path in $G$ from $x$ to $y$. If $G$ is not Hc, but $G+e$ is Hc for every $e \in E(\bar{G})$, then $G$ is said to be maximal nonhamiltonian-connected (MnHc).

If $X$ is a nonhamiltonian MnHc graph and $x y$ is any edge in $X$, then $X+e$ has a hamiltonian cycle containing $x y$ for each $e \in E(\bar{X})$. Thus we can replace each $X_{i}$ in the graphs depicted in Figures 11 and 12, with any nonhamiltonian MnHc graph, to obtain an MNT graph satifying the conditions of Theorem 3.12.

Z. Skupień brought to our attention that the Petersen graph, the Coxeter graph and the Isaacs' snarks $J_{k}$ for odd $k \geq 7$ are examples of nonhamiltonian MnHc graphs (see [9], [12] and [13]), so that the graphs $X_{i}$ can be chosen from these graphs, with $x$ and $y$ corresponding to two adjacent vertices in each $X_{i}$. Any $X_{i}$ may also be replaced by the middle block of a generalized sputnik, with $x$ and $y$ chosen as in Figure 10. Although the middle block of a generalized sputnik is not $\mathrm{MnHc}$, it does satisfy the condition required by the $G_{i}$ with respect to the chosen $x$ and $y$.

Next, we consider the case where $x$ and $y$ have the same neighbourhood.

Theorem 3.13 Let $G$ be a graph with a minimum vertex-cut $S=\{x, y\}$ such that $G-S$ consists of three noncomplete components $G_{1}, G_{2}, G_{3}$ and $N_{G_{i}}(x)=N_{G_{i}}(y)=N_{i} \neq V\left(G_{i}\right)$, for $i=1,2,3$.

Then $G$ is maximal nontraceable if and only if the following hold:

(i) $x y \in E(G)$.

(ii) $X_{i}=\left\langle V\left(G_{i}\right) \cup\{x\}\right\rangle$ is $M N H$ for $i=1,2,3$.

(iii) At most one of the graphs $\left\langle N_{i}\right\rangle$ has a universal vertex.

(iv) All three graphs $G_{i}$ are traceable and at least two of them are homogeneously traceable. 
(v) If $u$ is a universal vertex of $\left\langle N_{i}\right\rangle$ for some $i \in\{1,2,3\}$, then $G_{i}$ is traceable from $u$.

Proof. Suppose $G$ is MNT.

(i) As in the proof of Theorem 3.12 it follows that $x y \in E(G)$.

(ii) Let $u, v \in V\left(X_{2}\right)$; $u v \notin E(G)$. Then $G+u v$ has a hamiltonian path, $P$. If $X_{2}$ is hamiltonian, then $G_{2}$ has a hamiltonian path $Q$ with both endvertices in $N_{2}$. But then, replacing the subpath of $P$ in $G_{2}$ with $Q$, we obtain a hamiltonian path of $G$. This contradiction proves that $X_{2}$ is nonhamiltonian and that $G_{2}$ has no hamiltonian path with both endvertices in $N_{2}$. Similarly, for $i=1,3$ the graph $X_{i}$ is non-hamiltonian and $G_{i}$ does not have a hamiltonian path with both endvertices in $N_{i}$. This implies that $P$ has one endvertex in $G_{1}$ and the other in $G_{3}$, and hence $P$ has a subpath which is a hamiltonian path of $G_{2}+u v$, with both endvertices in $N_{2}$. Thus $X_{2}+u v$ is hamiltonian. Hence $X_{2}$ and, similarly, $X_{1}$ and $X_{3}$, are MNH. It also follows similarly that $Y_{i}=\left\langle V\left(G_{i}\right) \cup\{y\}\right\rangle$ is $\mathrm{MNH}$ for $i=1,2,3$.

(iii) Consider $G+v_{1} v_{2} ; v_{1} \in N_{1}, v_{2} \in N_{2}$. Since $G_{i}$ does not have a hamiltonian path with both endvertices in $N_{i} ; i=1,2,3$, it follows that a hamiltonian path $P$ in $G+v_{1} v_{2}$ has the structure shown in Figure 13, in which $G_{1}$ and $G_{2}$ can be interchanged.

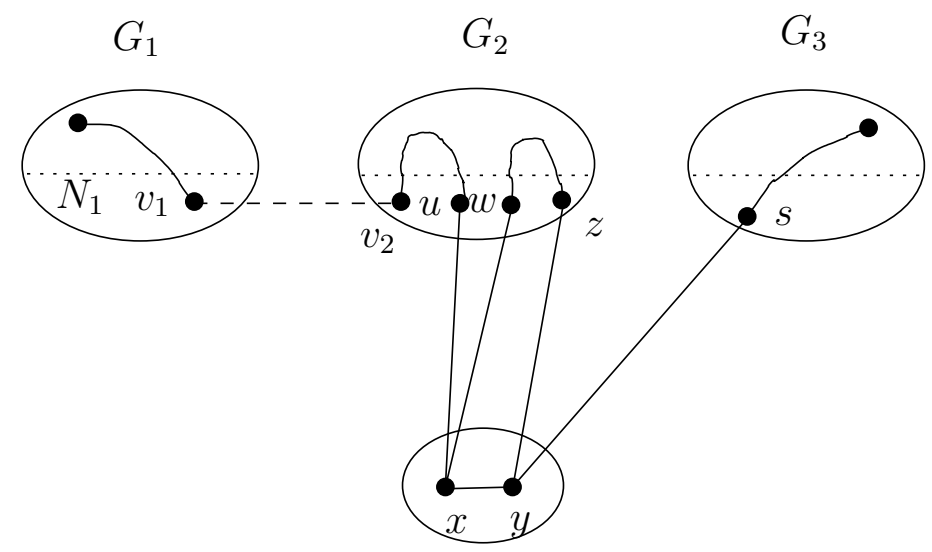

Figure 13: Sketch for Theorem 3.13

In the case shown, $G_{2}$ has a 2-path cover, $F^{1}\left[v_{2}, u\right], F^{2}[w, z]$, with $v_{2}, u, w, z \in N_{2}$.

Suppose $v_{i}$ is a universal vertex of $\left\langle N_{i}\right\rangle$, for $i=1,2$. Then $v_{2}$ is adjacent to $w$. If in $P$ we replace the subpath $P\left[v_{1}, z\right]$ with the path $v_{1} x F^{1}\left[u, v_{2}\right] F^{2}[w, z]$, then we obtain a hamiltonian path in $G$, which is a contradiction. This proves that, for any choice of $i$ and $j, i \neq j, i, j \in\{1,2,3\}$, at least one of $\left\langle N_{i}\right\rangle$ and $\left\langle N_{j}\right\rangle$ has no universal vertices. Hence at most one of the graphs $\left\langle N_{k}\right\rangle ; k=1,2,3$, has a universal vertex.

(iv) If $v \in V\left(G_{i}\right)-N_{i} ; i \in\{1,2,3\}$, then $G_{i}+v x$ has a hamiltonian path; hence $G_{i}$ is traceable from $v$. Suppose one of the $G_{i}$, say $G_{2}$, is not homogeneously traceable. 
Then there is a vertex $v_{2} \in N_{2}$ such that $G_{2}$ is not traceable from $v_{2}$. Now let $v_{1} \in N_{1}$. Then $G+v_{1} v_{2}$ has a hamiltonian path with the structure shown in Figure 13 and so $G_{1}$ has a hamiltonian path with $v_{1}$ as endvertex. Hence $G_{1}$ is homogeneously traceable. Similarly, $G_{3}$ is homogeneously traceable.

(v) Suppose one of the induced subgraphs $\left\langle N_{i}\right\rangle$, say $\left\langle N_{2}\right\rangle$, has a universal vertex $v_{2}$, but $G_{2}$ is not traceable from $v_{2}$. Then, as before, it follows that $G+v_{1} v_{2}$, where $v_{1} \in N_{1}$, has a hamiltonian path with the structure shown in Figure 13. As in the proof of (iii) it follows that $G$ has a hamiltonian path, a contradiction. Thus $G_{2}$ is traceable from $v_{2}$.

Conversely, suppose $G$ satisfies conditions (i)-(v). It follows from (ii) that $G_{i}$ has no hamiltonian path with both endvertices in $N_{i} ; i=1,2,3$, and hence $G$ is not traceable.

If $v \in V\left(G_{i}\right)-N_{i} ; i \in\{1,2,3\}$ then (ii) implies that $X_{i}+v x$ is hamiltonian, and hence has a hamiltonian path with $v$ and $x$ as endvertices. Thus each graph $X_{i}$ is traceable from each vertex in $G-N_{i}$ to $x$. Similarly, each graph $Y_{i}$ is traceable from every vertex in $G-N_{i}$ to $y$.

Now let $u, v \in V(G)$ and $u v \notin E(G)$. We show that $G+u v$ is traceable.

Case 1. $u, v \in V\left(G_{i}\right) \cup\{x, y\}$, say $i=2$.

From (ii) and our assumption that $N_{G_{i}}(x)=N_{G_{i}}(y)$ it follows that $\left\langle V\left(G_{i}\right) \cup\{x, y\}\right\rangle$ has a hamiltonian cycle containing the edge $x y$ and hence has a hamiltonian path $Q[x, y]$. Since $G_{1}$ is traceable to $x$ and $G_{3}$ is traceable from $y$, it follows that $G+u v$ is traceable.

Case 2. $u \in V\left(G_{i}\right)-N_{i}, v \in V\left(G_{j}\right)-N_{j}, i \neq j$, say $i=1$ and $j=2$.

$G_{1}$ is traceable from $u$, and $X_{2}$ is traceable from $v$ to $x$, and $G_{3}$ is traceable from $y$; hence $G+u v$ is traceable.

Case 3. $u \in N_{i}, v \in N_{j}, i \neq j$, say $i=1$ and $j=2$.

At least one of $G_{1}$ and $G_{2}$, say $G_{1}$, is homogeneously traceable. Hence $G_{1}$ has a hamiltonian path $P^{1}[a, u]$ for some $a \in V\left(G_{1}\right)$. If $v$ is not a universal vertex of $G_{2}$, let $w \in N_{2}$ such that $v w \notin E(G)$. Then, by (ii), $X_{2}+v w$ is hamiltonian and hence $X_{2}$ has a hamiltonian path $P^{2}[v, w]$. But $G_{3}$ has a hamiltonian path $P^{3}[y, z]$ for some $z \in V\left(G_{3}\right)$. The path $P^{1}[a, u] P^{2}[v, w] P^{3}[y, z]$ is a hamiltonian path of $G+u v$.

Now suppose $v$ is a universal vertex of $N_{2}$. In this case it follows from (v) that $G_{2}$ has a hamiltonian path $Q^{2}[b, u]$ for some $b \in V\left(G_{2}\right)$ and by (iii) $u$ is not a universal vertex of $G_{1}$, so $G_{1}$ has a hamiltonian path $P^{1}[u, t]$ for some $t \in N_{1}$. The path $Q^{2}[b, v] Q^{1}[u, t] x P^{3}[y, z]$ is a hamiltonian path of $G+u v$.

Case 4. $u \in N_{i}, v \in V\left(G_{j}\right)-N_{j}, i \neq j$, say $i=1$ and $j=2$.

If $u$ is a universal vertex of $\left\langle N_{1}\right\rangle$, then $G_{1}$ is traceable from $u$. If $u$ is not a universal vertex of $\left\langle N_{1}\right\rangle$, then, as shown in Case $3, X_{1}$ is traceable from $u$. In either case $G+u v$ has a hamiltonian path, $Y_{2}$ is traceable from $v$ to $y$ and $G_{3}$ is traceable from a vertex in $N_{3}$.

An example of a graph that satisfies the conditions of Theorem 3.13 is depicted in Figure 14. In this graph, $G_{1} \cong G_{2} \cong G_{3}$ is homogeneously traceable and $\left\langle V\left(G_{i}\right) \cup\{x\}\right\rangle$ 
is isomorphic to the Petersen graph, which is $\mathrm{MNH}$.

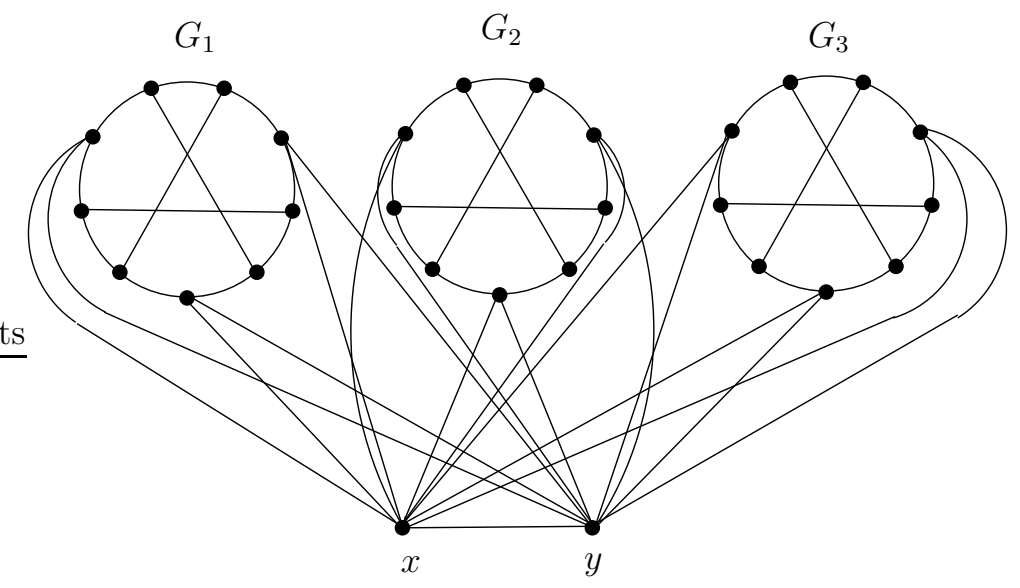

Figure 14: Graph that satisfies the conditions of Theorem 3.13

A graph $G$ is hypohamiltonian if $G$ is nonhamiltonian, but $G-v$ is hamiltonian for all $v \in V(G)$. A graph that is MNH as well as hypohamiltonian is called maximal hypohamiltonian $(M H H)$. We can replace any of the graphs $\left\langle V\left(G_{i}\right) \cup\{x\}\right\rangle$ in the graph depicted in Figure 14 by an MHH graph. (Then $G_{i}$ is hamiltonian, and hence homogeneously traceable.) Examples of MHH graphs are the Petersen graph, the Coxeter graph, Chisala's $G_{3}$-snark, and the Isaacs' snarks $J_{k}$ for odd $k \geq 5$ (see e.g. [1], [3], [4] and [11] for definitions).

Acknowledgement This paper contains results from the thesis of the third author which resulted from work done on toughness of MNT graphs at the Detour Workshops held at Salt Rock, South Africa in 2004 and 2006. The authors wish to thank the NRF and UNISA for funding the workshops.

\section{References}

[1] J.A. Bondy, Variations on the hamiltonian theme, Can. Math. Bull. 15 (1972), 57-62.

[2] F. Bullock, M. Frick and J. Singleton, Smallest claw-free, 2-connected, nontraceable graphs and the construction of maximal nontraceable graphs, Discrete Math. 307 (2007) 1266-1275.

[3] B.P. Chisala, On generating snarks, Discuss. Math. Graph Theory 18 (1998), 147158.

[4] L. Clark and R. Entringer, Smallest maximally nonhamiltonian graphs, Period. Math. Hung. 14 (1983), 57-68.

[5] L.H. Clark, R.C. Entringer and H.D. Shapiro, Smallest maximally nonhamiltonian graphs II, Graphs Comb. 8 (1992), 225-231. 
[6] A. Dudek, G.Y. Katona and A.P. Wojda, Hamiltonian Path Saturated Graphs with Small Size, Discrete App. Math. 154(9) (2006), 1372-1379.

[7] M. Frick and J. Singleton, Cubic maximal nontraceable graphs, Discrete Math. 307 (2007) 885-891.

[8] M. Frick and J. Singleton, Lower bound for the size of maximal nontraceable graphs, Electronic Journal of Combinatorics 12(1) (2005) R32.

[9] R. Kalinowski and Z. Skupień, Large Isaacs' graphs are maximally non-Hamiltonconnected, Discrete Math. 82 (1990) 101-104.

[10] R.C. Read and R.J. Wilson, An Atlas of Graphs, Oxford Science Publications, Oxford University Press, 1998.

[11] J.E. Singleton, Maximal Nontraceable Graphs, Ph.D. thesis, University of South Africa, Pretoria, 2005.

[12] Z. Skupien, On homogeneously traceable graphs and digraphs, 27 Internationales Wissenschtliches Kolloquium, Tech. Hochschule Ilmenau (GDR), 1982, Heft 5, 199-201.

[13] Z. Skupień, Maximally non-Hamilton-connected and hypohamiltonian graphs, in: M. Borowiecki and Z. Skupień, eds., Graphs, Hypergraphs and Matroids. III (Proc. Kalsk 1988 Conf), Higher Coll. of Eng. Zielona Góra, 1989, 133-144.

[14] B. Zelinka, Graphs maximal with respect to absence of hamiltonian paths, Discuss. Math. Graph Theory 18 (1998), 205-208. 